DAMTP-97-6

\title{
Cosmic microwave background polarization, Faraday rotation, and stochastic gravity-waves backgrounds
}

\author{
Massimo Giovannini] \\ DAMTP, Silver Street, Cambridge, CB3 9EW, United Kingdom
}

\begin{abstract}
A magnetic field, coherent over the horizon size at the decoupling and strong enough to rotate the polarization plane of the CMBR, can be generated from the electromagnetic vacuum fluctuations amplified by the space-time evolution of the dilaton coupling. The possible relevance of this result for superstring inspired cosmological models is discussed. Particular attention will be paid to the connection between Faraday rotation signals and stochastic gravity-wave backgrounds.
\end{abstract}

Accepted for publication in Physical Review D

\footnotetext{
${ }^{1}$ e-mail: M.Giovannini@damtp.cam.ac.uk
} 


\section{Introduction}

The polarization of the Cosmic Microwave Background Radiation (CMBR) represents a very interesting observable which has been extensively investigated in the past both from the theoretical [1] and experimental points of view [2]. Forthcoming satellite missions like MAP and PLANCK [3] seem to be able to achieve a level of sensitivity which will enrich decisively our experimental knowledge of the CMBR polarization with new direct measurements.

If the background geometry of the universe is homogeneous but not isotropic the CMBR is naturally polarized [1]. This phenomenon occurs, for example, in Bianchi-type I models [1]. On the other hand if the background geometry is homogeneous and isotropic (like in the Friedmann-Robertson-Walker [FRW] case) it seems very reasonable that the CMBR acquires a small degree of linear polarization provided the radiation field has a non-vanishing quadrupole component at the moment of last scattering [5].

Before decoupling photons, baryons and electrons form a unique fluid which possesses only monopole and dipole moments, but not quadrupole. Needless to say, in a homogeneous and isotropic model of FRW type a possible source of linear polarization for the CMBR becomes efficient only at the decoupling and therefore a small degree of linear polarization seems a firmly established theoretical option which will be (hopefully) subjected to direct tests in the near future. The discovery of a linearly polarized CMBR could also have a remarkable impact upon other (and related) areas of cosmology. Indeed the linear polarization of the CMBR is a very promising laboratory in order to directly probe the speculated existence of a large scale magnetic field (coherent over the horizon size at the decoupling) which might actually rotate (through the Faraday effect [6]) the polarization plane of the CMBR.

Consider, for instance, a linearly polarized electromagnetic wave of physical frequency $\omega$ travelling along the $\hat{x}$ direction in a cold plasma of ions and electrons together with a magnetic field $(\bar{B})$ oriented along an arbitrary direction ( which might coincide with $\hat{x}$ in the simplest case). If we let the polarization vector at the origin $(x=y=z=0, t=0)$

be directed along the $\hat{y}$ axis, after the wave has travelled a length $\Delta x$, the corresponding angular shift $(\Delta \alpha)$ in the polarization plane will be :

$$
\Delta \alpha=f_{e} \frac{e}{2 m}\left(\frac{\omega_{p l}}{\omega}\right)^{2}(\bar{B} \cdot \hat{x}) \Delta x
$$


(conventions: $\omega_{B}=e B / m$ is the Larmor frequency; $\omega_{p l}=\sqrt{4 \pi n_{e} e^{2} / m}$ is the plasma frequency $n_{e}$ is the electron density and $f_{e}$ is the ionization fraction ; we use everywhere natural units $\hbar=c=k_{B}=1$ ). It is worth mentioning that the previous estimate of the Faraday rotation angle $\Delta \alpha$ holds provided $\omega \gg \omega_{B}$ and $\omega \gg \omega_{p l}$. The quantity $\omega_{B} f_{e} \omega_{p l}^{2} \Delta x$ is also called Rotation Measure and, incidentally, the integrated version of Eq. (1.1) along the line of sight is one of the primary tools in the radio-astronomical measurements of the galactic magnetic field [17]. From Eq. (1.1) by stochastically averaging over all the possible orientations of $\bar{B}$ and by assuming that the last scattering surface is infinitely thin (i.e. that $\Delta x f_{e} n_{e} \simeq \sigma_{T}^{-1}$ where $\sigma_{T}$ is the Thompson cross section) we get an expression connecting the RMS of the rotation angle to the magnitude of $\bar{B}$ at $t \simeq t_{d e c}$

$$
\left\langle(\Delta \alpha)^{2}\right\rangle^{1 / 2} \simeq 1.6^{0}\left(\frac{B\left(t_{d e c}\right)}{B_{c}}\right)\left(\frac{\omega_{M}}{\omega}\right)^{2}, \quad B_{c}=10^{-3} \text { Gauss }, \quad \omega_{M} \simeq 3 \times 10^{10} \mathrm{~Hz}
$$

(in the previous equation we implicitly assumed that the frequency of the incident electromagnetic radiation is centred around the maximum of the CMBR). We can easily argue from Eq. (1.2) that if $B\left(t_{d e c}\right) \gtrsim B_{c}$ the expected rotation in the polarization plane of the CMBR is non negligible. Even if we are not interested, at this level, in a precise estimate of $\Delta \alpha$, we point out that more refined determinations of the expected Faraday rotation signal (for an incident frequency $\omega_{M} \sim 30 \mathrm{GHz}$ ) were recently carried out [7] leading to a result fairly consistent with (1.1). Then, provided a sizeable Faraday rotation is detected, the question which could immediately arise concerns the origin of such an intense field.

In homogeneous and isotropic cosmological models it seems not so obvious, theoretically, to justify the existence of a large scale magnetic field. In the context of the galactic magnetic field problem different mechanisms have been suggested in order to generate a field coherent at least over a (present) scale of $100 \mathrm{Kpc-1} \mathrm{Mpc.} \mathrm{These} \mathrm{mechanisms} \mathrm{generally} \mathrm{rely} \mathrm{either}$ upon inflationary models [8, 9, 10, 11] or upon the cosmological phase transitions (like the electro-weak phase transition [12] or the quark-hadron phase transition [13]). Recently [14] it was also observed that it is possible to use the $U_{Y}(1)$ anomaly to generate very energetic fields at the electroweak scale. It is generally difficult to generate directly the (inter)-galactic magnetic field and some of the above mentioned scenarios have necessarily to rely upon other (plasma physics) mechanisms (like the galactic dynamo or the anisotropic 
collapse mechanisms [15) able to inflate the initially small value of the "seed" 16] fields (i. e. $B \sim 10^{-24}-10^{-20}$ Gauss) up to the observed value of $10^{-6}$ Gauss at the galactic scale [17]. In this paper we are mainly interested in fields coherent at even larger scales (typically the decoupling scale) and we want to analyse the impact of a magnetic field generated in some string inspired model of cosmological evolution upon the Faraday rotation effect described by Eqs. (1.1)-(1.2).

In General Relativity (GR) the coupling of the gauge fields to the geometry is dictated by the equivalence principle, in String theory the unified value of the gravitational and gauge coupling (at the String scale) is provided instead by the dilaton field. Therefore in GR the gauge fields cannot be directly amplified thanks to the classical evolution of the background geometry since their equations of motion (in a four-dimensional conformally flat geometry) turn out to be invariant under the Weyl rescaling of the metric tensor. On the contrary in string inspired models the dilaton coupling does amplify the gauge fields as it was explicitly shown in a specific model [10] based on the pre-big-bang scenario [18]. Gauge fields can be also amplified during the relaxation of the dilaton towards the minimum of its potential providing non-trivial bounds on the value of the dilaton mass [11].

The question which we want to address here is whether a magnetic field as intense as $1-2 \times 10^{-3}$ Gauss (in short $B\left(t_{d e c}\right) \gtrsim B_{c}$ ) at the decoupling scale can naturally emerge thanks to the time evolution of the dilaton.

The plan of the paper is then the following. In Section 2 we will introduce the basic notions of String-inspired cosmological models discussing few theoretical assumptions which will be used all along the calculation of the amplification of the electromagnetic vacuum fluctuations in dilaton-driven and string driven-scenarios . Great attention will be paid on the role played by magnetic inhomogeneities coherent over the horizon size at decoupling in order to give an estimate of the Faraday rotation measure. In Section 3 we will point out that the fast growth of the dilaton field might also efficiently amplify gravity waves with a growing spectral amplitude and we will argue that the bounds usually applied on the stochastic gravity waves background might give complementary (and new!) constraints on the parameters of the models under study. Section 4 contains a summary of the main findings of this investigation and some concluding remarks. 


\section{Electromagnetic inhomogeneities}

One of the main features of the standard big-bang model is that the Universe started its evolution in a very hot, strongly coupled, highly curved state [19]. The low energy string theory effective action [20] (and its tree-level solutions [18) seem to motivate a picture where the initial state of the Universe instead of being hot and dense as in the standard cosmological context, was the string perturbative vacuum, namely a state with flat metric, vanishing gauge coupling $\left(g=e^{\phi / 2}=0, \phi=-\infty\right)$ and no matter content (except, perhaps, some very weakly interacting and highly diluted gas of fundamental strings).

The first assumption of this class of models is that the dynamics of the Universe can be consistently described in terms of the lowest order string effective action [20] in $(3+1)$ space-time dimensions :

$$
S=-\int d^{4} x \sqrt{-G} e^{-\phi}\left(R+\partial_{\mu} \phi \partial^{\mu} \phi+\frac{1}{4} F_{\mu \nu} F^{\mu \nu}-\frac{1}{12} H_{\mu \nu \alpha} H^{\mu \nu \alpha}\right)
$$

( $\phi$ controls the tree-level four-dimensional gauge coupling $g^{2}=e^{\phi} ; G_{\mu \nu} \equiv a^{2} \eta_{\mu \nu}$ is the four-dimensional metric which will be assumed to be conformally flat $\left[\eta_{\mu \nu}\right.$ is the usual Minkowski metric with signature $(+,-,-,-)] ; F_{\mu \nu}=\nabla_{[\mu} A_{\nu]} \equiv \partial_{[\mu} A_{\nu]}$ is the Maxwell field strength; $H_{\mu \nu \alpha}=\partial_{[\mu} B_{\nu \alpha]}$ is the antisymmetric tensor field strength).

We work in the String frame where the dilaton is directly coupled to the Einstein-Hilbert term and the string length $\lambda_{S}$ is truly a constant. The String and the more common Einstein frames are equivalent (up to a conformal transformation which redefines the dilaton field) only at tree-level but not when the higher order corrections in the string tension $\left(\alpha^{\prime}=\lambda_{S}^{-2}\right)$ are included and for this reason we prefer to perform our estimates, from the very beginning, in the String frame where the original expansions of the effective action (both in powers of $\alpha^{\prime}$ and in powers of $g$ ) are defined. One of the purposes of this paper is actually to show that starting only with the simplest non-trivial system containing the fewest number of relevant degrees of freedom (i.e. graviton and dilaton) it is possible to generate (by parametric amplification) the gauge fields and the antisymmetric tensor field.

This way of thinking corresponds to a kind of "minimality" assumption in the number of relevant degrees of freedom required in order to describe the evolution of the universe from its initial weakly coupled state. This assumption might be of course debatable since 
using different approaches it is perfectly possible to start from the very beginning with some classical configuration of antisymmetric tensor [21] or form fields [22].

On the other hand the minimality assumption allows already a reach set of phenomenological implications which might be tested in the future and then, before going to more complicated scenarios we want to explore if, in the present one, consistent phenomenological implications exist at all.

From the Eq. (2.1) we can deduce immediately that the rapid variation of the dilaton will excite not only scalar and tensor fluctuations (like in General Relativity) but also the gauge and antisymmetric tensor field fluctuations. If, according to our minimality assumption, the gauge fields are classically zero

$$
F_{\mu \nu} \equiv 0
$$

their quantum mechanical fluctuations $\left(A_{i}(k) \sim 1 / \sqrt{k}\right.$ in Fourier space) can be amplified thanks to the classical evolution of the dilaton field. The same phenomenon occurs in the case of the antisymmetric tensor field which we can also put consistently to zero

$$
H_{\mu \nu \alpha} \equiv 0
$$

and whose vacuum fluctuations can also be amplified by the classical evolution of the dilaton field.

In the following we will concentrate on the gauge fields which might have some effect upon the Faraday Rotation measurements and in order to start our program we want to present the low energy evolution equations of the dilaton field which can be obtained from Eq. (2.1) with the assumptions (2.2)-(2.3):

$$
\begin{aligned}
R_{\mu}^{\nu} & +\nabla_{\mu} \nabla^{\nu} \phi=0 \\
R & -\nabla_{\mu} \phi \nabla_{\nu} \phi G^{\mu \nu}+2 G^{\mu \nu} \nabla_{\mu} \nabla_{\nu} \phi=0
\end{aligned}
$$

( $\nabla$ is the Riemann covariant derivative; $R_{\mu}^{\nu}$ and $R$ are, respectively, the Ricci tensor and curvature scalar computed from the metric $G_{\mu \nu}$ ). Even if general solutions of the previous system of non-linear differential equations can be found in an anisotropic metric (with arbitrary number of spatial dimensions) of Bianchi-type I [23], we will mainly focus our attention, for sake of simplicity, on the case of an isotropic and spatially flat four-dimensional 
Friedmann-Robertson-Walker metric, where the equations of motion (2.4)-(2.5) can be rewritten as :

$$
\begin{aligned}
& \mathcal{H}^{\prime}-\mathcal{H}^{2}-\mathcal{H} \bar{\phi}^{\prime}=0 \\
& \bar{\phi}^{2}-3 \mathcal{H}^{2}=0
\end{aligned}
$$

(where $\bar{\phi}^{\prime}=\phi^{\prime}-3 \mathcal{H}$ ). We want to stress that the isotropy assumption of the present model might be also quite important for further possible discussion which go beyond the scope of the present paper. Namely it is not clear at all how the isotropy of the background might be achieved in the context of these models where the Weyl tensor of the background geometry is initially non-vanishing. It is well known that the process of particle production might make the Weyl tensor vanishing [25], but, at present, it is not clear at all if this mechanism might operate in the same way also in the context of string inspired models.

Notice that $\bar{\phi}$ is invariant under the scale factor duality 18 which is often invoked as one of the main theoretical motivations of the whole scenario. The duality symmetry implies that each solution of the low-energy equations of motion (2.6) might be related to another solution (of the same system of equations) whose physical properties can be different from the original one. The classical time evolution of the dilaton background might of course drive not only the classical evolution of the geometry but also the evolution of their fluctuations. Moreover the time evolution of the dilaton might also act as a "pump" field by amplifying the initially small (quantum) fluctuations of the other fields (like the string photon and the string axion) whose homogeneous part is exactly zero (see Eq. (2.2)-(2.3)). The scalar and tensor fluctuations of the background might either inherite the symmetries of the background or develop new symmetries [24] and perhaps something similar can happen for the axion field inhomogeneities [26] and for the electromagnetic inhomogeneities which we are going to specifically discuss and exploit in the present investigation.

The evolution equation for the Maxwell fields fluctuations will then become

$$
\partial_{\mu}\left(e^{-\phi} \sqrt{-G} F^{\mu \nu}\right)=0
$$

or, in Fourier space, for the two physical polarizations of the appropriate (canonically nor- 
malised) vector potentials

$$
A_{k}^{\prime \prime}+\left[k^{2}-V(\eta)\right] A_{k}=0, \quad V(\eta)=g\left(g^{-1}\right)^{\prime \prime}=\frac{\phi^{2}}{4}-\frac{\phi^{\prime \prime}}{2}
$$

(we wrote the previous equation using the radiation gauge condition $A_{0}=\bar{\nabla} \cdot \bar{A}=0$; the prime denotes the derivation with respect to conformal time $\eta$ while the over-dot denotes the derivation with respect to cosmic time $t$ ). In our context $V(\eta) \rightarrow 0$ for $\eta \rightarrow \pm \infty$ and then asymptotically Eq. (5) defines two Bunch-Davies vacua [25].

The effective potential barrier in Eq. (2.8) leads to wave amplification or, equivalently, to particle production. Indeed the positive frequency defining asymptotically the vacuum state to the left of the barrier $(\eta \rightarrow-\infty)$ will be in general a linear superposition of modes which are of positive and negative frequency with respect to the vacuum to the right $(\eta \rightarrow+\infty$ limit). The coefficients of the Bogoliubov transformation connecting the "left" and the "right" vacuum will determine the spectral distribution of the produced photons.

In order to compute the amplification factor we must use the explicit time evolution of the dilaton background suggested by the inflationary models based upon the String theory low energy effective action [18]. The solutions of the evolution equations derived in Eqs. (2.6) seem to motivate a picture where the universe starts in a cold and empty state ( the dilaton perturbative vacuum with $g=0, \phi=-\infty$ and $H=\dot{a} / a=0$ ) which is not stable towards small dilaton perturbations. The unstable dilaton background starts growing $(\dot{\phi}>0)$ at the same rate of the curvature $(\dot{H}>0)$.

During this phase of increasing coupling and curvature the background is practically driven by the dilaton kinetic energy. The dilaton-driven phase can be described in terms of the lowest order string theory effective action only up to a time $\eta=\eta_{s}$ when the curvature reaches the string scale $H_{s} \sim \lambda_{s}^{-1} \sim \sqrt{\alpha^{\prime}}$. Provided $g_{s}=g\left(\eta_{s}\right) \ll 1$ the higher orders in $g$ can be safely neglected. On the contrary for $\eta>\eta_{s}$ the expansion in $\alpha^{\prime}=\lambda_{S}^{-2}$ breaks down and all the higher orders in $\alpha^{\prime}$ should be taken into account. From $\eta_{s}$ up to $\eta_{r}$ the background enters then a stringy phase whose unknown duration $\left(z_{s}=\eta_{s} / \eta_{r}\right)$ represents a free parameter of the whole scenario. As extensively discussed in the past it seems very hard (if not impossible [27]) to have a graceful exit to the ordinary FRW decelerated phase $(\dot{a}>0$, $\ddot{a}<0, \phi\left(\eta_{r}\right)=\phi_{r}=$ const.) without taking into account a stringy phase driven by the higher 
$\alpha^{\prime}$ corrections (which should be included in the original String frame and not in the Einstein frame as originally [28] suggested). Recently different examples in $(1+1),(3+1)$ and $(d+1)$ dimensions [29] seemed to show that either the back-reaction effects or the first $\alpha^{\prime}$ correction might regularize the curvature also slowing down the dilaton growth. The impact of the $\alpha^{\prime}$ corrections can be also important for the evolution equation describing the propagation of tensor modes (see Sec. 3) which will receive, in principle, also the contribution of the higher derivatives appearing in the modified action. Whenever the minimality constraint (2.3) is enforced it is possible to write the action in the String frame in such a way that the quadratic curvature corrections appear in the well known Gauss-Bonnet combination. In this specific case no higher derivatives are expected in the equations of motion of the tensor fluctuations.

In the low energy phase $\left(\eta<\eta_{s}\right)$ the dilaton coupling is known exactly and we have, solving Eqs. (2.6):

$$
a(\eta) \simeq|\eta|^{-\frac{1}{\sqrt{3}+1}}, \quad \phi=-\sqrt{3} \ln |\eta|+\text { const. }, \quad \eta<\eta_{S} .
$$

During the stringy phase the average time evolution of the dilaton field might be described by:

$$
a(\eta) \sim \eta^{-1}, \quad \phi=-2 \beta \ln |\eta|+\text { const. }, \quad \beta=-\frac{\phi_{\mathrm{s}}-\phi_{\mathrm{r}}}{2 \ln \mathrm{z}_{\mathrm{s}}}, \quad \eta_{\mathrm{s}}<\eta<\eta_{\mathrm{r}} .
$$

Finally for $\eta>\eta_{r}$ the background is dominated by radiation

$$
a(\eta) \simeq \eta, \quad \phi=\phi_{r}=\text { const. }, \quad \eta>\eta_{r} .
$$

By assuming, in our case, the initial states of the Maxwell field (for $\eta \rightarrow-\infty$ ) correspond to the Bunch-Davies "conformal" vacuum [25] we can write the general solution of Eq. (2.8) for each mode $A_{k}$ in the three temporal region as

$$
\begin{aligned}
& A_{k}(u)=\frac{1}{\sqrt{k}} \sqrt{u} H_{\nu}^{(1)}(u), \quad \nu=\frac{\sqrt{3}-1}{2}, \quad \eta<\eta_{s} \\
& A_{k}(u)=\frac{1}{\sqrt{k}} \sqrt{u}\left[D_{+} H_{\mu}^{(2)}(u)+D_{-} H_{\mu}^{(1)}(u)\right], \quad \mu=\frac{|2 \beta-1|}{2}, \quad \eta_{s}<\eta<\eta_{r} \\
& A_{k}(u)=\frac{1}{\sqrt{k}}\left[c_{+} e^{i u}+c_{-} e^{-i u}\right], \quad \eta>\eta_{r}
\end{aligned}
$$


(where $u=k \eta ; H^{(1)}$ and $H^{(2)}$ denote the Hankel functions [30] of first and second kind; notice that for $|\eta| \rightarrow \infty, \sqrt{u} H^{(2,1)}(u) \rightarrow e^{\mp i k \eta} / \sqrt{k}$ the minus and plus sign corresponds, respectively, to $H^{(2)}$ and $\left.\left.H^{(1)}\right]\right)$.

The Bogoliubov coefficients determined by matching the previous solutions (and their first derivatives) in $\eta=\eta_{s}$ and $\eta=\eta_{r}$ will then be:

$$
\begin{aligned}
& \left|c_{-}\right| \simeq\left|k \eta_{r}\right|^{-\beta}, \quad k_{s}<k<k_{r} \\
& \left|c_{-}\right| \simeq\left|k \eta_{s}\right|^{\frac{1}{2}-\frac{\sqrt{3}}{2}}\left|k \eta_{r}\right|^{-\frac{1}{2}}\left|\eta_{r} / \eta_{s}\right|^{-\beta+\frac{1}{2}}, \quad k<k_{s} .
\end{aligned}
$$

Notice that in the first of the two previous equations we have chosen, implicitly, $\beta>1 / 2$. The reason of this choice will be clear in the following. We can anyway mention that the only sizeable effects associated with models with $\beta<1 / 2$ occurs in practice for $\beta<0$. This case might be of course possible from the purely mathematical point of view but it would correspond to a dynamical situation which is rather peculiar, namely the case where the dilaton background decreases already during the stringy phase. All the present indications concerning the dynamics of the stringy phase come from the study of higher curvature corrections to the low-energy effective action (2.1), and in this framework it seems only possible, at the moment, that the dilaton is linearly increasing (in cosmic time) implying $\beta>0$.

In the background model defined in Eqs. (2.9)-(2.10)-(2.11) $V(\eta)$ grows like $\eta^{-2}$ for $\eta \rightarrow$ $0^{-}$in the dilaton driven phase and reaches its maximal value during the stringy phase (around $\eta=\eta_{r}$ ) going rapidly to zero for $\eta>\eta_{r}$. Therefore modes with $u_{r} \lesssim 1$ will remain under the barrier during the whole stringy phase. The coefficients determined in this "sudden" approximation lead, in general, to an ultraviolet divergence in $\left|c_{-}\right|^{2}$ (which is related, in a second quantisation context, to the number of produced photons). The reason is that, for modes with $u \gtrsim 1$ the sudden approximation beaks down and the potential step in Eq. (2.8) should be replaced by a smooth function. In this way we find indeed, according to the standard treatment [25], that $\left|c_{-}\right|^{2}$ is exponentially suppressed for all the modes with $u_{r} \gtrsim 1$ so that particle production will be ignored for the purpose of this paper.

Different modes will go under the barrier (crossing the horizon) at different times and 
the energy density of the amplified fluctuations will then be given by

$$
\frac{d \rho_{B}}{d \ln \omega}=\frac{\omega^{4}}{\pi^{2}}\left|c_{-}\right|^{2}
$$

where $\omega=k / a$ is the physical frequency which we will always express at the present time. We also define

$$
r(\omega)=\frac{1}{\rho_{\gamma}} \frac{d \rho_{B}}{d \ln \omega}=\frac{\omega^{4}}{\rho_{\gamma}} \frac{\left|c_{-}\right|^{2}}{\pi^{2}}, \quad \rho_{\gamma}(t)=M_{P}^{2} H_{r}^{2}\left(\frac{a_{r}}{a}\right)^{4} \equiv \omega_{r}^{4}\left(\frac{g_{r}}{4 \pi}\right)^{2}
$$

which measures the fraction of electromagnetic energy stored in the mode $\omega$. Note that $\omega_{r} \sim$ $a_{r} / \eta_{r}=\sqrt{g_{r} / 4 \pi} 10^{11} \mathrm{~Hz}$ is the maximal amplified frequency red-shifted today and $g_{r}=e^{\phi_{r} / 2}$ is the coupling at the end of the stringy phase which could typically range between $10^{-1}$ and $10^{-3}$ 31], also that $g_{r} / 4 \pi \simeq \sqrt{H_{r} / M_{P}}$. The quantity $r(\omega)$ is quite useful since it stays constant during both the radiation and matter dominated epochs when the conductivity of the universe is reasonably high [6, 32]. For modes $\omega>\omega_{s}=\omega_{r} / z_{s}$ crossing the horizon during the stringy phase we can thus obtain the spectrum from the first expression in Eq. (2.13)

$$
r(\omega) \simeq\left(\frac{g_{r}}{4 \pi}\right)^{2}\left(\frac{\omega}{\omega_{r}}\right)^{4-2 \beta}, \quad \omega_{s}<\omega<\omega_{r} .
$$

For modes crossing the horizon during the dilaton driven phase we have instead (from the second expression in Eq. (2.13)):

$$
r(\omega)=\left(\frac{\omega}{\omega_{r}}\right)^{4-\sqrt{3}} z_{s}^{-\sqrt{3}}\left|\eta_{s} / \eta_{r}\right|^{2 \beta}, \quad \omega<\omega_{s}
$$

(in the last formula $\left|\eta_{s} / \eta_{r}\right|^{2 \beta}=\left(g_{s} / g_{r}\right)^{-2}$ from Eq. (2.10)).

Since, as we stressed at the very beginning, we are dealing here with homogeneous and isotropic models of background evolution we have to require that $r(\omega)<1$ for all the frequencies. This implies, during the stringy phase $\beta<2$ (or using Eq. (2.10) $\ln g_{s} / g_{r}<$ $\left.-2 \ln z_{s}\right)$. The same bound can be obtained for modes crossing the horizon during the dilaton-driven phase. In order to have a sizeable rotation in the polarization plane of the CMBR we have also to require from Eq. (1.2)

$$
B\left(t_{d e c}\right) \gtrsim B_{c}
$$


at the decoupling scale which is also equivalent, in our notation, to

$$
r\left(\omega_{\text {dec }}\right) \gtrsim 7.5 \times 10^{-8}, \quad \omega_{\text {dec }} \sim 10^{-16} \mathrm{~Hz}
$$

(having used for the decoupling temperature $T_{d e c} \simeq 0.25 \mathrm{ev}$ with $\left.\rho_{\gamma}\left(t_{d e c}\right) \simeq\left(\pi^{2} / 15\right) T_{\gamma}^{4}\right)$.

If the decoupling scale crossed the horizon during the dilaton driven-phase the magnetic energy can fulfil the critical density bound and the Faraday rotation condition (2.19) provided

$$
-2 \log _{10} z_{s} \lesssim \log _{10} \frac{g_{s}}{g_{r}} \lesssim-0.86 \log _{10} z_{s}-27.05-0.56 \log _{10} g_{r} / 4 \pi
$$

which corresponds to a narrow range in the parameter space centred around $\log _{10} z_{s} \simeq 25$ and $\log _{10} g_{s} / g_{r} \lesssim-50$.

If the decoupling scale crossed the horizon during the stringy phase the inequality Eq. (2.19) requires instead:

$$
\beta \gtrsim \frac{100.87}{54+\log _{10} g_{r} / 4 \pi} .
$$

Since $g_{r} / 4 \pi \sim 10^{-1}-10^{-3}, \beta \gtrsim 1.9$. We notice that this range of values of $\beta$ would correspond to a flat (or slightly "blue") spectrum of electromagnetic fluctuations which crossed the horizon during the stringy phase. For example for $\beta \sim 1.91-1.92$ and $g_{r} / 4 \pi \sim 0.1$ we would get $B\left(t_{d e c}\right) \simeq 1.5-2 \times 10^{-3}$.

It could seem that the variation of $\beta$ is really so tiny to be irrelevant but, on the other hand we see from Eq. (2.10) that a small variation in $\beta$ translates in a larger variation both in the duration of the stringy phase and in its average coupling constant. At the same time if $\beta<3 / 2$ the spectrum will become steeper and, asymptotically, violet. In other words for $\beta \ll 3 / 2$ the stringy branch of the spectrum $\left(\omega_{s}<\omega<\omega_{r}\right)$ will become more and more similar to the dilaton driven branch $\left(\omega<\omega_{s}\right)$ with sharply increasing slopes.

We want finally to stress that the calculations presented in this paper do not consider a very interesting and new effect pointed out by Olesen [33] who discussed the possibility of an inverse cascade in the magneto-hydrodynamical (MHD) evolution of the amplified fields. The Olesen's argument is quite general and based on scaling properties of the MHD equations in $(3+1)$-dimensions and it was also explicitely investigated in the context of $(2+1)$ dimesional MHD simulations [34]. If the energy spectrum of the primordial magnetic field is steep enough an inverse cascade can occur and small scale magnetic fields will coalesce giving 
rise, ultimately, to a magnetic field of smaller amplitude but bigger coherence scale (see Ref. [34] for an explicit shell model of $(3+1)$-dimensional MHD cascade). It is interesting to point out that the Olesen scaling argument might be applied also to our spectra with the result that an inverse cascade is very likely to occur also in the present case. It is then possible to speculate that at large scales there will be more power than the one we estimated. Our bounds might be then different if the inverse cascade effect will be properly discussed. This observation is even more relevant for the calculations of microwave background anisotropies produced by a stochastic background of magnetic fields where the inclusion of MHD cascades is decisive in order to make any definite prediction beyond the ones discussed in [10].

\section{Gravitational versus Magnetic Inhomogeneities}

The same background evolution leading to the amplification of gauge fields according to the mechanism discussed in the previous Section might lead to the amplification of tensor modes which will turn, after they reenter the horizon, into a stochastic gravity-waves background. Before claiming any physical effect, what we should always do, in the framework of any particular model, is to compare the regions of parameter space allowed (or excluded) by the amplification of the vacuum fluctuations of different fields. In principle only the overlaps of the allowed regions for the amplification of the diverse inhomogeneities will represent a viable theoretical framework which should be ultimately compared with the available experimental data. The purpose of the present Section is to compute the gravity-wave background produced in the same class of models examined in Sec. 2 and to compare it with the present upper bounds and/or future planned sensitivities of gravity-waves detectors specifically designed for the study of stochastic sources. Needless to say that our present knowledge of gravity-waves background is by no means direct. Even if stochastic backgrounds of gravity waves are still not detected there are at least three very useful theoretical bounds and various experimental upper limits coming from operating devices. By defining the energy density of gravity-waves in critical units

$$
\Omega_{G W}(\omega)=\frac{1}{\rho_{\gamma}} \frac{d \rho_{G W}}{d \ln \omega}
$$


we have that, on theoretical ground, there are at least three constraints coming form large and intermediate scales [35]. At large scales the most stringent bound comes from the high degree of isotropy of the CMBR radiation which imposes

$$
\Omega_{G W}(\omega)<7 \times 10^{-11} h_{100}^{-2}\left(\frac{\omega}{\omega_{0}}\right)^{2}, \omega \sim \omega_{0}
$$

$\left(\omega \sim \omega_{0}=3.2 \times 10^{-18} h_{100} \mathrm{~Hz}\right.$, where $h_{100}=H_{0} /\left(100 \mathrm{~km} \mathrm{sec}^{-1} \mathrm{Mpc}^{-1}\right)$ is the present uncertainty on the value of the Hubble parameter; in this section we will always express the energy density of the gravity-waves background at the present time). At intermediate scales another bound comes from pulsar timing measurements

$$
\Omega_{G W}(\omega)<10^{-8}, \quad \omega \sim 10^{-8} \mathrm{~Hz}
$$

A further (indirect) bound comes finally from the standard nucleosynthesis analysis imposing

$$
h_{100}^{2} \int \Omega_{G W}(\omega) d \ln \omega \lesssim 0.5 \times 10^{-5}
$$

Recently the Rome group [36] produced an experimental upper limit ( $\Omega_{G W} \lesssim 500$ for $\omega \sim$ $1 \mathrm{KHz}$ ) on the existence of stochastic gravity-waves backgrounds using cryogenic bar detectors. A sensitivity of $\Omega_{G W}(\omega \sim 1 \mathrm{KHz}) \sim 10^{-4}$ is expected. Resonant spherical detectors [37] offer a foreseen sensitivity of $\Omega_{G W}(\omega \sim 1 \mathrm{KHz}) \sim 10^{-7}$, whereas the LIGO-VIRGO expected sensitivity [35, 38, 39] is $\Omega_{G W}\left(\omega_{L}\right) \sim 10^{-10}$ for $\omega_{L} \sim 100 \mathrm{~Hz}$ (to be compared with the initial operating sensitivity of $\Omega_{G W}\left(\omega_{L}\right) \sim 10^{-5}$ ).

In Ref. [39] the LIGO sensitivity was carefully compared to the expected signal coming from string inspired models of cosmological evolution. The authors of [39], however, completly ignored the possible theoretical constraints coming from the amplification of electromagnetic inhomogeneities [10]. Our attitude is that some of these theoretical constraints are more stringent than the direct ones, as we will implicitly show and as it was also (incidentally) pointed out in previous theoretical works on the subject [41].

The game in this Section will to compute the gravity-waves signal produced by the same background evolution examined in Sec. 3. We will then compare the signal with the expected sensitivity of interferometric detectors, and we will then contrast the allowed regions of parameter space in principle accessible to the LIGO-VIRGO planned sensitivity with the 
regions of parameter space allowing for a detectable Faraday rotation of the CMBR(in the hypothesis the CMBR is polarized). Of course different regions of the parameter space will be excluded and the question will be to decide which are the allowed regions.

The linearized version of the evolution equation (2.4) we are interested in can be easily obtained by perturbing the metric for pure tensor modes

$$
G_{\mu \nu} \rightarrow G_{\mu \nu}+h_{\mu \nu}, G^{\mu \nu} h_{\mu \nu}=0, \quad \nabla_{\mu} h_{\nu}^{\mu}=0
$$

with the result that

$$
\square h_{i}^{j}-\dot{\phi} h_{i}^{j}=0
$$

(where $\square=G^{\mu \nu} \nabla_{\mu} \nabla_{\mu}$ and the dot denotes the derivative with respect to the cosmic time coordinate). In terms of the eigenstates of the Laplace-Beltrami operators

$$
\nabla^{2} h_{i}^{j}(k)=-k^{2} h_{i}^{j}(k)
$$

Eq. (3.6) becomes, in cosmic time :

$$
\ddot{h_{i}^{j}}-\dot{\bar{\phi}} \dot{h_{i}^{j}}+\omega^{2} h_{i}^{j}=0
$$

(notice that the driving term in the evolution equation of the tensor modes depends only on the term $\bar{\phi}$ which is invariant under scale factor duality). The evolution equation for the canonically normalized tensor modes of oscillation reads

$$
\mu^{\prime \prime}+\left[k^{2}-V(\eta)\right]=0, \quad V(\eta)=\left(\frac{g}{a}\right)\left(\frac{a}{g}\right)^{\prime \prime} \equiv \frac{a^{\prime \prime}}{a}+\frac{\phi^{\prime 2}}{4}-\frac{\phi^{\prime \prime}}{2}-\mathcal{H} \phi^{\prime}
$$

(where $h_{i}^{j}=a^{-1} \mu e_{j}^{i}$ and $e_{i}^{j}$ label the two physical polarization of the gravitational wave in vacuum). We can immediately notice that the wave equation for the Fourier modes of the canonically normalized vector potentials examined in Sec. 3 is indeed very similar to this equation. By inserting the background solutions discussed in Eqs. (2.9)-(2.10)-(2.10) we get again a general solution (in the three temporal regions) which can be expressed in terms of Hankel functions whose indices are anyway very different from the ones of Sec. 3. By matching in the transitions points the values of $\mu$ and $\mu^{\prime}$ we can get again the bogoliubov coefficients giving the amplification of the tensor modes. The Bogoliubov coefficients computed for tensor modes in the background given by Eqs. (2.9)-(2.10)-(2.11) are reported 


\begin{tabular}{|c|c|c|}
\hline & $k_{\text {dec }}<k<k_{s}$ & $k_{s}<k<k_{r}$ \\
\hline Gravitons & $\left|k \eta_{r}\right|^{-1 / 2} z_{s}^{3 / 2}\left(g_{s} / g_{r}\right)^{-1} \ln k \eta_{s}$ & $\left|k \eta_{r}\right|^{-1 / 2-|3-2 \beta| / 2}$ \\
\hline Photons & $\left|k \eta_{r}\right|^{-1 / 2} z_{s}^{-1 / 2}\left(g_{s} / g_{r}\right)^{-1}$ & $\left|k \eta_{r}\right|^{-\beta}$ \\
\hline
\end{tabular}

Table 1: We report the Bogoliubov coefficients for obtained by studying the amplification of electromagnetic inhomogeneities (photons) and tensor inhomogeneities (gravitons) in the minimal model of dilaton evolution discussed in Sec. 2. The same model leads to different amplifications coefficients since the normal modes of the electromagnetic fluctuations are only coupled to the dilaton. The normal modes of oscillation of tensor fluctuations are instead coupled to the dilaton and to the geometry.

in Table 1 where, for comparison, we also report the Bogoliubov coefficients computed in Sec. 2 for the same background evolution but for electromagnetic fluctuations. Since this estimate is straightforward and can be easily reproduced by repeating the algebra outlined in Sec. 2 and by using, this time, Eq. (3.9), we will not report the details of the calculation. From the Bogoliubov coefficients it is immediate to get the energy density of the amplified gravitons expressed in critical units

$$
\Omega_{G W}(\omega)=\frac{1}{\rho_{\gamma}} \frac{\omega^{4}}{\pi^{2}}\left|c_{-}\right|^{2}
$$

which becomes, using the results listed in Table 1

$$
\begin{aligned}
& \Omega_{G W}(\omega)=z_{\text {dec }}^{-1} g_{s}^{2}\left(\frac{\omega}{\omega_{s}}\right)^{3} \ln \left(\frac{\omega_{s}}{\omega}\right), \quad \omega_{\text {dec }}<\omega<\omega_{s} \\
& \Omega_{G W}(\omega)=g_{r}^{2} z_{d e c}^{-1}\left(\frac{\omega}{\omega_{r}}\right)^{3-|3-2 \beta|}, \quad \omega_{s}<\omega<\omega_{r}
\end{aligned}
$$

$\left(z_{\text {dec }}=a / a_{e q} \simeq 10^{4}\right.$ takes into account the transition from radiation to matter dominance at $\left.\eta=\eta_{\text {dec }}\right)$.

The obtained gravity-wave spectral energy density consists of two branches: one ranging from $\omega_{\text {dec }}$ up to $\omega_{s}=\omega_{r} / z_{s}$ (the so-called "dilaton-driven" branch) and the other ranging from $\omega_{s}$ up to $\omega_{r}$ (the so-called "stringy" branch). To be precise we should also take into account the third branch of the spectrum which is the "matter" branch. In fact the transition from a 
radiation dominated epoch to a matter-dominated epoch does not amplify electromagnetic inhomogeneities but does certainly amplify the tensor modes. The spectral energy density in the "matter" branch (ranging from $\omega_{0}$ to $\omega_{\text {dec }}$ ) will be further suppressed compared to the spectrum in the dilaton-driven branch by the typical factor $\left(\omega_{d e c} / \omega\right)^{2}$. The COBE bound combined with the pulsar timing measurements and with the standard nucleosynthesis bound constrain the spectral slope during the stringy phase to be quite steep and namely we get :

$$
0<\beta<3
$$

The resulting spectral energy density shows then a quite sharp peak around $\omega_{r} \sim \mathrm{GHz}$ [28, 41]. The requirement that the produced gravity-wave background will be also detectable by the improved sensitivity of the gravity wave interferometers will instead imply, at the LIGO-VIRGO scale:

$$
\Omega_{G W}\left(\omega_{L}\right)>10^{-10}, \quad \omega_{L} \sim 10^{2} \mathrm{~Hz}
$$

If the LIGO-VIRGO scale went out of the horizon during the dilaton-driven phase this would imply that $\omega_{L}<\omega_{s}$, which would also imply $z_{s}<10^{9}$ (namely a short stringy phase). As we discussed in the previous Section such a short duration of the stringy phase is not compatible with the occurrence of a magnetic field strong enough to rotate the polarization plane of the CMBR, since we should have, at least $\omega_{\text {dec }}>\omega_{s}$ which means $z_{s}>10^{27}$ (since the decoupling scale is much larger that the LIGO-VIRGO scale). For this reason we will concentrate our attention on the case in which the LIGO-VIRGO scale went out of the horizon during the stringy phase (namely $z_{s}>10^{9}$ ). In this case the requirement (3.13) implies using the second of Eqs. (3.11):

$$
\begin{array}{ll}
\beta<\left(\frac{1}{3}+\frac{1}{9} \log _{10} \frac{g_{r}}{4 \pi}\right), & \beta<\frac{3}{2} \\
\beta>\left(\frac{8}{3}-\frac{1}{9} \log _{10} \frac{g_{r}}{4 \pi}\right), & \beta>\frac{3}{2}
\end{array}
$$

If the conditions expressed by Eq. (2.21) and by Eqs. (3.14)-(3.15) would be simultaneously satisfied we would have a range of parameters in our model which would allow both a sizeable Faraday Rotation and a signal detectable by LIGO-VIRGO. From the exclusion plots reported in Fig. $\mathbf{1}$ we see that this is indeed the case for a narrow slice of values of 
$\beta>3 / 2$

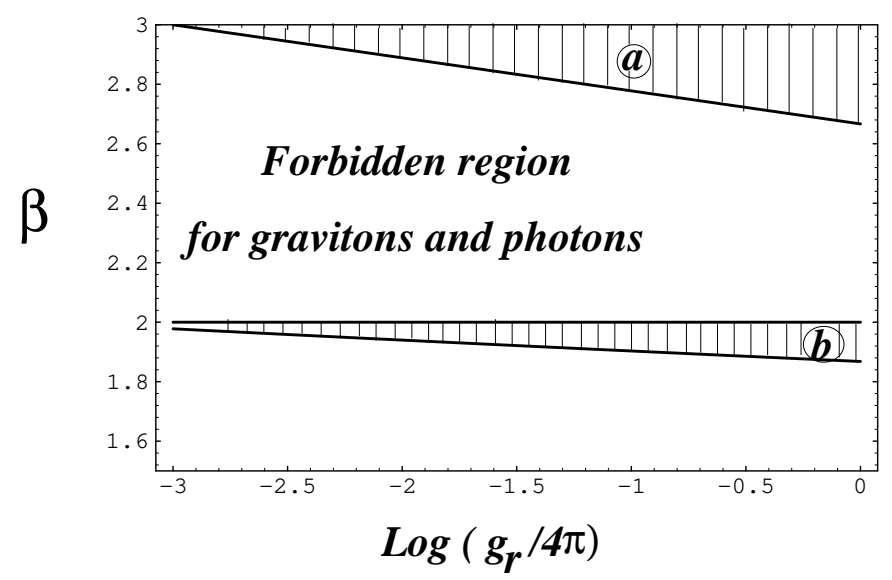

$\beta<3 / 2$

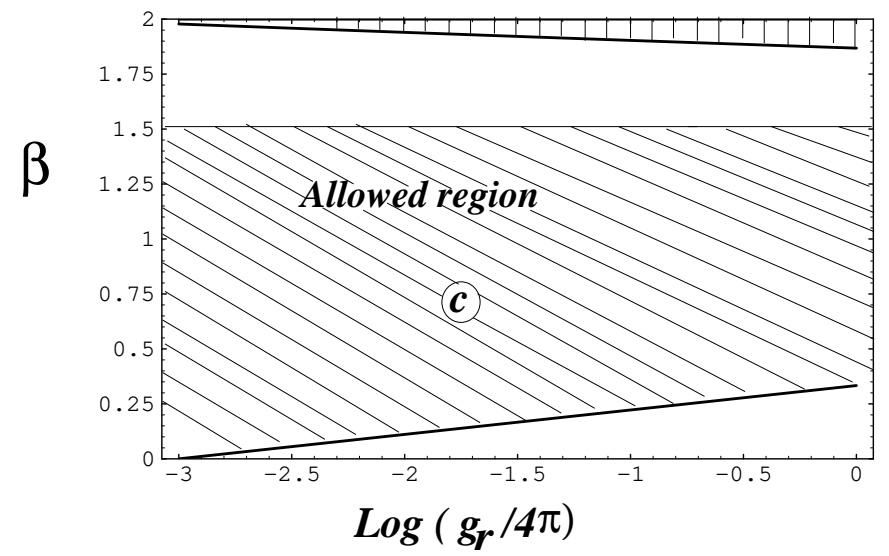

Figure 1: We plot the three different regions defining the interplay between gravitational-waves interferometry and Faraday Rotation measurements for the minimal models of dilaton evolution outlined in the present analysis. Regions a and $\mathbf{c}$ exclude a simultaneous detection of a strong gravity-waves background peaked in the $\mathrm{GHz}$ region and of a sizeable Faraday Rotation (in the hypothesis of the polarization of the CMBR). Region $\mathbf{b}$ allows for such an overlap of signals. We used the expected sensitivities of LIGO-VIRGO interferometers operating in the $10^{2} \mathrm{~Hz}$ region. 
$\beta$ (i. e. $1.7<\beta<2$ ). In Fig. 1 we plot $\beta$ versus the coupling constant at the end of the stringy phase which could typically range between $10^{-3}$ and $10^{-1}$ 40. We can see that the range of $\beta$ allowing only for a detectable stochastic gravity-waves background is wider that the region allowing also for a detectable Faraday Rotation of the CMBR. the very simple conclusions we can draw from these pictures is that if a signal will ever be detected by the advanced LIGO-VIRGO for this will most likely exclude any Faraday Rotation signal in the same range of parameters. A measurable Faraday Rotation is still compatible (even if in a narrow range) with a gravity wave background strongly peaked in the $\mathrm{GHz}$ region. We can finally notice that the allowed region for the detection of a stochastic background extends also for $\beta>2$ which is strictly forbidden by the closure density bound applied to the electromagnetic fluctuations amplified by the same dilaton evolution.

Our exclusion plots were obtained by using the foreseen sensitivity of the interferometers operating in the $10^{2} \mathrm{~Hz}$ region. Given the physical properties of the gravity-waves background we are discussing it could seem more appropriate, in principle, to use devices operating at even higher frequencies. Microwave cavities [43] with improved sensitivities seem a very promising option in this framework.

If the CMBR will turn out to be polarized and if a sizeable Faraday rotation will not be detected a stringy phase of low $\beta(\lesssim 3 / 2)$ will be definitely the most appealing option.

If $z_{s}<10^{9}$ (stringy phase even shorter) the interplay among Faraday Rotation measurements and stochastic gravity-waves backgrounds is irrelevant. Moreover the LIGO-VIRGO bound will imply, using the first of Eqs. (3.11), and up to logarithmic corrections

$$
z_{s}^{-3} g_{r}^{2}\left(\frac{g_{r}}{g_{s}}\right)^{2}>10^{21}
$$

Taking any value of $g_{r}$ the closure density bound applied on the amplified electromagnetic inhomogeneities would imply $\left(g_{r} / g_{s}\right)<z_{s}^{2}$ which is in sharp contradiction with the requirement (3.16). In this last case the elctromagnetic and tensor inhomogeneities are mutually excluding each other. 


\section{Discussion and Conclusions}

If a reasonable Faraday rotation is not detected, implying the absence of a strong magnetic field at the decoupling, this would reduce the parameter space of the minimal model of background evolution discussed in this paper definitely pointing towards $\beta<3 / 2$. It could become then interesting to discuss explicitly a stringy phase of "low $\beta$ ". It would also be important to compute precisely the magnitude of the Faraday Rotation due to a string cosmological magnetic field in order to compare it with the possible angular shift of the polarization plane of the CMBR produced, for example, by the galactic magnetic field itself red-shifted backwards in time up to the decoupling scale. If the galactic magnetic field would be purely the result of causal (i.e. plasma physics) mechanisms operating inside the galaxy after its formation without any pre-existing seed, it is unlikely to be present at decoupling. In this last scenario the detection of a sensible Faraday rotation would be a test for the primordial origin of the galactic field.

Another test concerning the possible primordial nature of the galactic field would come indeed from nucleosynthesis. It is actually well known that a magnetic field coherent over the horizon size at nucleosynthesis could have a significant impact on the abundances of the light nuclei making the process of their formation intrinsically anisotropic. There are fairly

precise bounds [44] (recently revisited [45]) on anisotropic nucleosynthesis implying a bound for the magnetic field strength

$$
B\left(t_{d e c}\right) \lesssim 0.1 \text { Gauss }
$$

(we expressed this condition at the decoupling for comparison; notice that in Ref. [45] this bound has been relaxed by one order of magnitude since it has been realized that the leading effects of a magnetic field at nucleosynthesis are connected with a change in the expansion rate giving, in our case $B\left(t_{d e c}\right) \lesssim 1$ Gauss). Nucleosynthesis bounds are then quite mild in our scenario since they would imply

$$
r\left(\omega_{\text {dec }}\right) \lesssim 7.5 \times 10^{-4}
$$

which is certainly satisfied since the $r\left(\omega_{\text {dec }}\right)$ required in order to rotate the polarization plane of the CMBR is typicallly three-four orders of magnitude smalller than the nucleosynthesis bound. 
All the super-string inspired models discussed in the present paper might also give rise to a stochastic background of gravity waves characterised by a strong peak in the $G H z$ frequency band [41, 42]. The range of parameters for which a strong peak can be produced has anyway a quite narrow overlap with the range of parameters producing a Faraday rotation. Therefore depending upon $z_{s}$ and $g_{s}$ it could be hard to have both the effects for all the parameter space. In particular the area in which a signal could be detected with interferometers extends also for $\beta>2$ (strictly forbidden by the critical density bound applied to the amplified electromagnetic fluctuations analysed here). This result of course holds only for the minimal model presented in this investigation and provided we treat perturbations in the linear regime. To relax one of this two hypothesis could change also drastically the conclusions. Another uncertainty in the estimates of the stochastic gravity-waves background might come from the dynamics of the internal dimensions and in particular (as shown in 42]) from the internal gradients. In any case it is amusing that, in the present context, Faraday rotation measurements and microwave cavities might give complementary constraints upon $\beta$ and ultimately, upon the duration of the stringy phase. Whether the dilaton-amplified electromagnetic fluctuations might be of some relevance for structure formation (as suggested in [10) is quite debatable but the detection of a Faraday rotation in the CMBR could give important clues by ruling out all but few of the theoretically possible models we discussed. If a sizeable Faraday Rotation will not be detected only a low $\beta$ stringy phase could give rise to a detectable gravity waves spectrum. It migh also turn out (from PLANCK observations) that the CMBR is not polarized. In this last case all the present investigation will be uninteresting, since without a polarized CMBR also the Faraday effect will not take place leaving the size of the magnetic field at the decoupling only constrained by the critical density bound and by the nucleosynthesis bounds.

\section{Acknowledgements}

I am very grateful to N. Turok for the stimulating environment which partially motivated this investigation and to A. C. Davis for helpful comments. I am indebted to M. Shaposhnikov for many important discussions on the various roles of the magnetic fields at the 
electroweak scale and I would also like to acknowledge M.Gasperini and G. Veneziano for previous collaboration. I thank E. Picasso for very interesting insights concerning microwave cavities.

\section{References}

[1] M. Rees, Astrophys. J. 153, L1 (1968).

[2] P. M. Lubin and G. F. Smoot, Astrophys. J. 245, 1 (1981); P. M. Lubin, P. Melese and G. F. Smoot, Astrophys. J. Lett. 273, L51 (1983).

[3] M. Bersanelli et. al. COBRAS/SAMBA Report of Phase A study Esa D/Sci(96)3; MAP home page http://map.gsfc.nasa.gov/

[4] E. Milaneschi and R. Fabbri, Astron. Astrophys. 151, 7 (1985).

[5] D. Coulson, R. Crittenden and N. G. Turok, Phys. Rev. D 52, 5402 (1995); Phys. Rev. Lett. 73, 2390 (1994).

[6] E. N. Parker, Cosmical Magnetic Fields ( Clarendon, Oxford, 1979); Y. B. Zeldovich, A. A. Ruzmaikin and D. D. Sokoloff, Magnetic Fields in Astrophysics (Gordon and Breach, New York, 1983).

[7] A. Kosowsky and A. Loeb, Astrophys. J 461, 1 (1996); D. Harari, J. Hayward and M. Zaldarriaga, Phys. Rev. D 55 (1997), in press.

[8] M. S. Turner and L. M. Widrow,Phys. Rev. D 37, 2743 (1988).

[9] B. Rathra, Astrophys. J. Lett. 391, L1 (1992).

[10] M. Gasperini, M. Giovannini and G. Veneziano, Phys. Rev. Lett. 75, 3796 (1985); Phys. Rev. D 52, 6651 (1995). 
[11] M. Giovannini, DAMTP-97-54, Phys. Rev. D (1997) (to appear).

[12] T. Vachaspati, Phys. Lett. B265, 258 (1991).

[13] J. M. Quashnock, A. Loeb and D. N. Spergel, Astrophys. J. 344, L49 (1989); B. Cheng and A. V. Olinto, Phys. Rev. D 50, 2421 (1994).

[14] M. Joyce and M. Shaposhnikov, Primordial magnetic fields, right electrons, and the abelian anomaly, CERN-TH-97-31, e-Print Archive: astro-ph/9703005.

[15] S. I. Vainstein and Ya B. Zeldovich, Sov. Phys. Usp. 15, 159 (1972); R. M. Kulsrud and S. W. Anderson, Astrophys. J. 396, 606 (1992).

[16] E. R. Harrison, Phys. Rev. Lett. 30, 188 (1973).

[17] M. J. Rees, Quart. J.R.A.S. 28, 197 (1987); P.P. Kronberg, Rep. Prog. Phys. 57, 325 (1994).

[18] G. Veneziano, Phys. Lett. B265, 287 (1991); M. Gasperini and G. Veneziano, Astropart. Phys. 1, 317 (1993).

[19] S. Weinberg, Gravitation and Cosmology, (Wiley, New York, 1972).

[20] C. Lovelace, Phys. Lett. B135, 75 (1984); E. S. Fradkin and A. A Tseytlin, Nucl. Phys. B261, 1 (1985); C. G. Callan et al., Nucl. Phys. B262, 593 (1985).

[21] E.J. Copeland, A. Lahiri, D. Wands, Phys.Rev.D 51, 1569 (1995); Phys.Rev.D 50, 4868 (1994).

[22] A. Lukas, B. A. Ovrut, D. Waldram, Phys.Lett. 393B, 65 (1997).

[23] M. Gasperini and G. Veneziano, Mod. Phys. Lett. A 8, 3701, 1993.

[24] M. Gasperini and M. Giovannini, Class. Quant. Grav. 14, 735 (1997).

[25] N. D. Birrel and P. C. W. Davies, Quantum Fields in Curved Space, (Cambridge University Press, Cambridge, England, 1982). 
[26] E.J. Copeland, Richard Easther, David Wands, SUSSEX-TH-97-001, e-Print Archive hep-th/9701082 (unpublished).

[27] R. Brustein and G. Veneziano, Phys. Lett. B329, 429 (1994); N. Kaloper, R. Madden, and K. Olive, Nucl. Phys. B452, 677 (1995); Phys. Lett. B371, 34 (1996).

[28] M. Gasperini and M. Giovannini, Phys. Lett. B287, 56 (1992).

[29] I. Antoniadis, J. Rizos and K. Tamvakis, Nucl. Phys. B415, 497 (1994); S. Y. Rey, Phys. Rev. Lett. 77, 1929 (1996); M. Gasperini and G. Veneziano, Phys. Lett. B387, 715 (1996); M. Gasperini, M. Maggiore and G. Veneziano, CERN-TH/96-267 (unpublished).

[30] M. Abramowitz and I. A. Stegun, Handbook of Mathematical Functions, (Dover, New York, 1972).

[31] V. Kaplunovsky, Phys. Rev. Lett. 55, 1036 (1985).

[32] A. C. Davis, K. Dimopoulos, Phys. Lett. B390, 87 (1996).

[33] P. Olesen, Phys.Lett. B398, 321 (1997).

[34] A. Brandenburg, K. Enqvist and P. Olesen, Phys.Lett. B392, 395 (1997); Phys.Rev. D

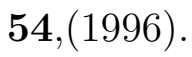

[35] K. S. Thorne, in 300 Years of Gravitation, edited by S. W. Hawking and W. Israel (Cambridge University Press, Cambridge, England, 1987).

L. P. Grishchuk, Sov. Phys. Usp. 31, 940 (1988);

B. Allen, in Proceedings of the Les Houches School on Astrophysical Sources of Gravitational Waves, edited by J. Marck and J.P. Lasota (Cambridge University Press, Cambridge England, 1996).

[36] P. Astone, G.V. Pallottino and G. Pizzella, LNF-96-001-IR (1996).

[37] S. Vitale, M. Cerdonio, E. Coccia and A. Ortolan, Phys. Rev. D 55, 1741 (1997). 
[38] A. Abramovici et al., Science 256, 325 (1992);

C. Bradaschia et al., Nucl. Instrum. Meth. A289, 518 (1990).

[39] B. Allen and R. Brustein, Detecting gravitational radiation from string cosmology with LIGO, WISC-MILW-96-TH-34, BGU-PH-96/09, e-Print Archive gr-qc/9609013 (unpublished).

[40] V. Kaplunovsky, Phys. Rev. Lett. 55, 1036 (1995).

[41] R. Brustein, M. Gasperini, M. Giovannini and G. Veneziano, Phys. Lett. B361, 45 (1995); M. Gasperini, M. Giovannini, Phys. Lett. B282, 36 1992; M.Gasperini, M. Giovannini, Phys. Rev. D 47, 1519 (1993)

[42] M. Giovannini, Phys. Rev. D 55, 595 (1997).

[43] F. Pegoraro, E. Picasso, and L. Radicati, J. Phys. A11, 1949 (1978).

[44] B. Cheng, D. N. Schramm and J. Truran, Phys. Rev. D 49, 5006 (1994); D. Grasso and H. Rubinstein, Astropart. Phys.3, 95 (1995).

[45] B. Cheng, A. V. Olinto D. N. Schramm and J. Truran, Phys. Rev. D 54, 4714; D. Grasso and H. Rubinstein, Phys. Lett. B379, 74 (1996); P. Kernan, G. Starkman and T. Vachaspati, Phys. Rev. D 54, 7207 (1996). 